\title{
THE USE OF GEOCENTRIC VARIABLES TO SEARCH FOR METEOROID STREAMS AND THEIR PARENTS
}

\author{
CL. FROESCHLÉ \\ Observatoire de la Côte d'Azur, B.P. 4229, F-06304 Nice, France \\ T.J. JOPEK
}

Obserwatorium Astronomiczne Uniwersytetu A. Mickiewicza, Sloneczna 36, PL-60286

Poznań, Poland

and

\section{G.B. VALSECCHI}

I.A.S. - Planetologia, via Fosso del Cavaliere, I-00133 Roma, Italy

\begin{abstract}
A set of geocentric variables suitable for the identification of meteoroid streams has been recently proposed and successfully applied to photographic meteor orbits. We describe these variables and the secular invariance of some of them, and discuss their use to improve the search for meteoroid stream parents.
\end{abstract}

Key words: Meteors, Meteoroid streams

\section{Introduction}

Valsecchi et al. (1999) have recently proposed a new orbital similarity criterion for meteor orbits rather different from the one proposed by Southworth and Hawkins in 1963 and widely used for meteoroid stream identifications ever since.

The criterion by Southworth and Hawkins (1963) is a generalized measure of distance in the 5-dimensional space of the orbital elements $q, e, i, \omega$ and $\Omega$ :

$$
\begin{aligned}
D_{S H}^{2}= & {\left[e_{2}-e_{1}\right]^{2}+\left[q_{2}-q_{1}\right]^{2}+\left[2 \sin \frac{I_{21}}{2}\right]^{2}+} \\
& {\left[\left(\frac{e_{2}+e_{1}}{2}\right)\left(2 \sin \frac{\pi_{21}}{2}\right)\right]^{2} }
\end{aligned}
$$

where

$$
\left[2 \sin \frac{I_{21}}{2}\right]^{2}=\left[2 \sin \frac{i_{2}-i_{1}}{2}\right]^{2}+\sin i_{1} \sin i_{2}\left[2 \sin \frac{\Omega_{2}-\Omega_{1}}{2}\right]^{2}
$$

and

$$
\pi_{21}=\omega_{2}-\omega_{1}+2 \arcsin \left[\cos \frac{i_{2}+i_{1}}{2} \sin \frac{\Omega_{2}-\Omega_{1}}{2} \sec \frac{I_{21}}{2}\right] .
$$

The formulation of $D_{S H}$, however, does not take into account that meteoroids are observed at heliocentric distance $r \simeq 1 \mathrm{AU}$, so that approximately either

$$
1=\frac{a\left(1-e^{2}\right)}{1+e \cos (-\omega)}
$$


or

$$
1=\frac{a\left(1-e^{2}\right)}{1+e \cos \left(180^{\circ}-\omega\right)}
$$

depending on whether the meteoroid is at the ascending or at the descending node of its orbit. This implies that although $D_{S H}$ is defined in a 5-dimensional space, meteoroids are actually observed only when they are on a suitable 4-dimensional hypersurface. Moreover the observational errors, in the passage from the observed quantities (time of fall, geocentric velocity and radiant coordinates) to the orbital elements, are redistributed among the latter in a very complicated way. The improved formulations of Southworth and Hawkins' criterion introduced by Drummond (1981) and Jopek (1993) have retained the same basic features, as they also are based on the orbital elements.

Valsecchi et al. (1999) described a set of geocentric quantities that characterize completely a meteor orbit, and introduced an orbital similarity criterion that, when applied to the best photographic meteor orbits, gives significantly different results from $D_{S H}$ in the case of low-inclination streams (Jopek et al. 1999). The geocentric quantities and the criterion based on them are described in Section 2; some of these quantities have near-invariance properties against secular perturbations, as shown in Section 3. The use of these properties to look for parent bodies of meteoroid streams is discussed in Section 4; the conclusions (Section 5) then follow.

\section{Geocentric Quantities and the Criterion Based on Them}

The following treatment is based on the geometric setup of Öpik's theory of close encounters (Öpik 1976; Carusi et al. 1990).

Let us assume that the Earth moves on an unperturbed circular orbit, of radius equal to 1, lying on the ecliptic and that the massless meteoroid is on an orbit, with orbital parameters $a, e, i, \omega$ and $\Omega$, which hits that of the Earth in one of the nodes. Putting the constant of gravity and the mass of the Sun equal to 1, and imposing that the heliocentric velocity of the Earth is $v_{\oplus}=1$, we have that the geocentric velocity of the meteoroid when crossing the Earth's orbit is:

$$
U=\sqrt{3-\bar{T}}
$$

where $T$ is the Tisserand parameter:

$$
T=\frac{1}{a}+2 \sqrt{a\left(1-e^{2}\right)} \cos i \text {. }
$$

In a reference frame centred on the Earth, with the $z$-axis perpendicular to the plane of the ecliptic, the $y$-axis in the direction of the Earth's velocity and the $x$-axis pointing away from the Sun, the unperturbed geocentric encounter velocity 
U of the meteoroid has components:

$$
\begin{aligned}
& U_{x}=U \sin \theta \sin \phi \\
& U_{y}=U \cos \theta \\
& U_{z}=U \sin \theta \cos \phi
\end{aligned}
$$

where $\theta$ is the angle between $\mathbf{U}$ and the $y$-axis, and $\phi$ is the angle between the $y$ - $z$ plane and that containing $\mathbf{U}$ and the $y$-axis. If the encounter is at the ascending node $-90^{\circ}<\phi<90^{\circ}$, while at the descending node $90^{\circ}<\phi<270^{\circ}$ ); moreover, if the meteoroid is receding from perihelion, $0^{\circ}<\phi<180^{\circ}$, and if it is approaching it $180^{\circ}<\phi<360^{\circ}$.

The components of $\mathrm{U}$ can be expressed in terms of $a, e$ and $i$ :

$$
\begin{aligned}
& U_{x}= \pm \sqrt{2-\frac{1}{a}-a\left(1-e^{2}\right)} \\
& U_{y}=\sqrt{a\left(1-e^{2}\right)} \cos i-1 \\
& U_{z}= \pm \sqrt{a\left(1-e^{2}\right)} \sin i
\end{aligned}
$$

and $a, e$ and $i$ in terms of the components of $\mathbf{U}$ :

$$
\begin{aligned}
a & =\frac{1}{1-U^{2}-2 U_{y}} \\
e & =\sqrt{U^{4}+4 U_{y}^{2}+U_{x}^{2}\left(1-U^{2}-2 U_{y}\right)+4 U^{2} U_{y}} \\
i & =\arctan \frac{U_{z}}{1+U_{y}} ;
\end{aligned}
$$

in equation (11) the minus sign must be used for pre-perihelion encounters, and in equation (13) for encounters at the descending node.

A set of variables suitable for our purposes is then composed of the modulus of the unperturbed geocentric velocity $U$, the two angles $\theta$ and $\phi$, given by

$$
\begin{aligned}
& \theta=\arccos \frac{U_{y}}{U} \\
& \phi=\arctan \frac{U_{x}}{U_{z}}
\end{aligned}
$$

and of the longitude of the Earth $\lambda_{\oplus}$ at the time of the meteor observation. These quantities are all directly observed, as $\theta$ and $\phi$ define the direction opposite to the geocentric radiant, in the frame moving with the Earth (and not that of fixed stars).

In practice, one obtains $U, \theta$ and $\phi$, through the components $U_{x}, U_{y}, U_{z}$, from the geocentric velocity $V_{G}$ and the equatorial coordinates of the meteor radiant $\alpha_{G}$ and $\delta_{G}$, by means of the expression:

$$
\left(\begin{array}{l}
U_{x} \\
U_{y} \\
U_{z}
\end{array}\right)=\hat{\mathbf{r}}(\lambda) \cdot \hat{\mathbf{p}}(\varepsilon) \cdot \frac{V_{G}}{29.7}\left(\begin{array}{l}
-\cos \delta_{G} \cos \alpha_{G} \\
-\cos \delta_{G} \sin \alpha_{G} \\
-\sin \delta_{G}
\end{array}\right)
$$


where $\hat{\mathbf{p}}(\varepsilon), \hat{\mathbf{r}}(\lambda)$ are rotational matrices around the $x$ - and $z$-axis, respectively, and the angle $\varepsilon$ denotes the inclination of the ecliptic plane to the celestial equator.

The new orbital similarity criterion $D_{N}$ introduced by Valsecchi et al. (1999) is given by:

$$
D_{N}^{2}=\left[U_{2}-U_{1}\right]^{2}+w_{1}\left[\cos \theta_{2}-\cos \theta_{1}\right]^{2}+\Delta \xi^{2}
$$

where

$$
\begin{aligned}
\Delta \xi^{2} & =\min \left[w_{2} \Delta \phi_{I}^{2}+w_{3} \Delta \lambda_{I}^{2}, w_{2} \Delta \phi_{I I}^{2}+w_{3} \Delta \lambda_{I I}^{2}\right] \\
\Delta \phi_{I} & =\left[2 \sin \frac{\phi_{2}-\phi_{1}}{2}\right] \\
\Delta \phi_{I I} & =\left[2 \sin \frac{180^{\circ}+\phi_{2}-\phi_{1}}{2}\right] \\
\Delta \lambda_{I} & =\left[2 \sin \frac{\lambda_{2}-\lambda_{1}}{2}\right] \\
\Delta \lambda_{I I} & =\left[2 \sin \frac{180^{\circ}+\lambda_{2}-\lambda_{1}}{2}\right]
\end{aligned}
$$

and $w_{1}, w_{2}, w_{3}$ are suitably defined weighting factors; $\Delta \xi$ is small either if both $\phi_{1}-\phi_{2}$ and $\lambda_{1}-\lambda_{2}$ are small, or if they are both close to $180^{\circ}$. In this case, the two meteors belong to the two showers corresponding to the two node crossings of essentially the same orbit.

Jopek et al. (1999) applied the new criterion, setting the three weighting factors equal to 1, to a set of 865 precisely measured photographic meteor orbits. They grouped the meteors by a single neighbour linking algorithm (Lindblad 1971) not requiring any a priori knowledge of stream orbits, and determined thresholds for $D_{N}$ by comparison with random samples having the same marginal distributions of the variables (Jopek and Froeschle 1997), in order to have a reliability level of 99\%. For comparison, they made also a classification using $D_{S H}$.

They found that $D_{S H}$ and $D_{N}$ give similar results for streams of moderate to high inclination, with $D_{N}$ often adding a few more members to some streams; on the other hand, for near-ecliptical streams the results of the two criteria were markedly different. In particular, 2 streams were identified only with $D_{S H}$ (the $\sigma$ Leonids and the Andromedids), while 5 were identified only with $D_{N}$ (the $\varepsilon$ Geminids, the Monocerotids, the $\alpha$ Virginids, the Northern $\delta$ Aquarids and the $\varepsilon$ Piscids).

Arithmetic mean orbital and geocentric data concerning the 15 streams identified by Jopek et al. (1999) with $D_{N}$ are given in Table I.

\section{Secular Invariance Properties of $U$ and $\cos \theta$}

Over not too long timescales, and in absence of planetary close encounters, one can assume that mostly secular perturbations affect meteoroid orbits; the most 


\section{TABLE I}

Arithmetic mean orbital and geocentric data of the streams found by Jopek et al. (1999) using $D_{N}$ amongst 865 precise photographic meteor orbits; $i, \omega$ and $\Omega$ are given for B1950.0. All angles are given in degrees, $q$ is in $\mathrm{AU}$, and $V_{G}$ in $\mathrm{km} / \mathrm{s}$. For streams identified as single ones, but possessing both a Northern and a Southern branch, the data are given separately for each branch.

\begin{tabular}{l|ccrrr|rrr}
\hline Stream name & \multicolumn{1}{c}{$e$} & \multicolumn{1}{c}{$i$} & \multicolumn{1}{c}{$\omega$} & \multicolumn{1}{c}{$\Omega$} & $V_{G}$ & \multicolumn{1}{c}{$\theta$} & $\phi$ \\
\hline Lyrids & 0.92 & 0.99 & 80 & 214 & 32 & 47 & 119 & 198 \\
$\alpha$ Capricornids (N) & 0.58 & 0.78 & 6 & 268 & 134 & 23 & 89 & 262 \\
$\alpha$ Capricornids (S) & 0.63 & 0.62 & 4 & 89 & 329 & 18 & 89 & 276 \\
Perseids & 0.95 & 0.95 & 113 & 150 & 139 & 59 & 139 & 164 \\
$\kappa$ Cygnids & 0.98 & 0.76 & 39 & 202 & 147 & 25 & 88 & 194 \\
$\varepsilon$ Geminids & 0.81 & 0.96 & 174 & 231 & 203 & 70 & 166 & 258 \\
Taurids (N) & 0.32 & 0.85 & 3 & 299 & 213 & 30 & 104 & 267 \\
Taurids (S) & 0.34 & 0.82 & 6 & 118 & 27 & 28 & 104 & 275 \\
Quadrantids & 0.98 & 0.68 & 72 & 171 & 282 & 41 & 116 & 176 \\
Geminids & 0.14 & 0.90 & 24 & 324 & 261 & 35 & 117 & 258 \\
$\chi$ Orionids(N) & 0.38 & 0.83 & 3 & 291 & 265 & 28 & 101 & 267 \\
$\chi$ Orionids(S) & 0.51 & 0.79 & 5 & 96 & 77 & 25 & 93 & 276 \\
Monocerotids & 0.18 & 1.00 & 37 & 129 & 80 & 43 & 112 & 286 \\
Leonids & 0.98 & 0.92 & 162 & 173 & 235 & 71 & 170 & 168 \\
$\sigma$ Hydrids & 0.24 & 0.98 & 126 & 122 & 78 & 58 & 137 & 295 \\
Orionids & 0.57 & 0.97 & 165 & 83 & 29 & 66 & 155 & 288 \\
$\alpha$ Virginids (N) & 0.41 & 0.83 & 8 & 288 & 34 & 28 & 99 & 263 \\
$\alpha$ Virginids (S) & 0.32 & 0.87 & 7 & 118 & 198 & 31 & 103 & 275 \\
S. $\delta$ Aquarids & 0.08 & 0.97 & 27 & 151 & 308 & 41 & 118 & 278 \\
N. $\delta$ Aquarids & 0.10 & 0.95 & 21 & 328 & 142 & 38 & 117 & 262 \\
$\varepsilon$ Piscids (N) & 0.58 & 0.76 & 5 & 268 & 190 & 22 & 89 & 263 \\
$\varepsilon$ Piscids (S) & 0.61 & 0.73 & 4 & 85 & 5 & 21 & 88 & 276 \\
Draconids & 1.00 & 0.70 & 25 & 177 & 196 & 17 & 71 & 175 \\
Cyclids & 0.83 & 0.12 & 3 & 119 & 26 & 3 & 108 & 296 \\
$\alpha$ Pegasids & 0.97 & 0.68 & 7 & 200 & 230 & 11 & 42 & 226 \\
\hline
\end{tabular}

important among them is the one related to the cycle of $\omega$ (Kozai 1962). Assuming that all the planets are on circular coplanar orbits, and that the small body orbit is far from mean motion and secular resonances, it leaves invariant, besides the Kozai integral $K$ (Thomas and Morbidelli 1996), the $z$-component of the orbital angular momentum

$$
L_{z}=\sqrt{a\left(1-e^{2}\right)} \cos i
$$

and the orbital energy

$$
E=-\frac{1}{2 a} \text {. }
$$

But then

$$
T=\frac{1}{a}+2 \sqrt{a\left(1-e^{2}\right)} \cos i=2\left(L_{z}-E\right)
$$


is constant, and therefore so is $U$. Finally, if $a$ and $U$ are conserved, so is

$$
\cos \theta=\frac{1-U^{2}-1 / a}{2 U} .
$$

To illustrate the invariance of $U$ and $\cos \theta$ over significant time scales, we have integrated for $100000 \mathrm{yr}$ a fictitious object on an orbit similar to that of $96 \mathrm{P} / \mathrm{Mach}$ holz 1, in a simplified solar system consisting only of Jupiter and the Earth, both on circular coplanar orbits. The results are shown in Fig. 1, where are given the time behaviours of, from top to bottom, $\Omega, \omega, i, e, q, U$ and $\cos \theta$. The ordinate range shown for each variable is the total range allowed to an Earth-crossing meteoroid on a non-hyperbolic orbit; the top five panels show that the variables entering $D_{S H}$ $(q, e, i, \omega$ and $\Omega$ ) undergo large oscillations, spanning in some cases the entire allowed range, whereas the variations of $U$ and $\cos \theta$, in the two lowermost panels are small compared to their allowed ranges. Quantitatively, we have that, in the time span integrated, $0.015 \leq q \leq 1.517 \mathrm{AU}, 0.533 \leq e \leq 0.995,10^{\circ} .86 \leq i \leq 82^{\circ} .22$, while $1.390 \leq U \leq 1.527$ and $-0.5481 \leq \cos \theta \leq-0.4457$.

Valsecchi et al. (1999) propose to exploit the secular near-invariance of $U$ and $\cos \theta$ by combining them into a reduced criterion:

$$
D_{R}^{2}=\left[U_{2}-U_{1}\right]^{2}+w_{1}\left[\cos \theta_{2}-\cos \theta_{1}\right]^{2}
$$

where, as before, $w_{1}$ is a weighting factor. This criterion can be useful to find parent candidates for meteor showers with orbits having different $q, e, i, \omega$ and $\Omega$, due to secular perturbations on one and the same meteoroid stream (Babadzhanov and Obrubov 1993), but one must keep in mind that it gives only a necessary, not a sufficient, condition for such identifications, that must be validated by detailed modelling of the stream evolution.

\section{Using Geocentric Variables to look for Stream Parents}

In their application of $D_{N}$ to photographic meteor orbits, Jopek et al. (1999) did not relate the streams that they found to the parent bodies. When the orbit of the parent is very similar to that of the stream, this is a straightforward task; things are not so simple when the orbit of the candidate parent body has evolved away from the node-crossing condition.

We have taken the data of Table I and removed the streams composed of only two members (Draconids, Cyclids and $\alpha$ Pegasids), and the single Southern $\alpha$ Capricornid, due to the low significance of average quantities in these cases; we have then applied the reduced criterion of Eq. (30), with weight factor equal to unity. We set as threshold $D_{R} \leq 0.12$ and, to reduce the number of spurious associations, we stipulated that the difference between the longitudes of perihelion $\varpi=\omega+\Omega$ of the stream and of the candidate parent body should not exceed $17^{\circ}$. These thresholds were chosen in order to recover, as a possible parent body, comet 96P/Machholz 1 for the Quadrantids (this association is not widely accepted, as 


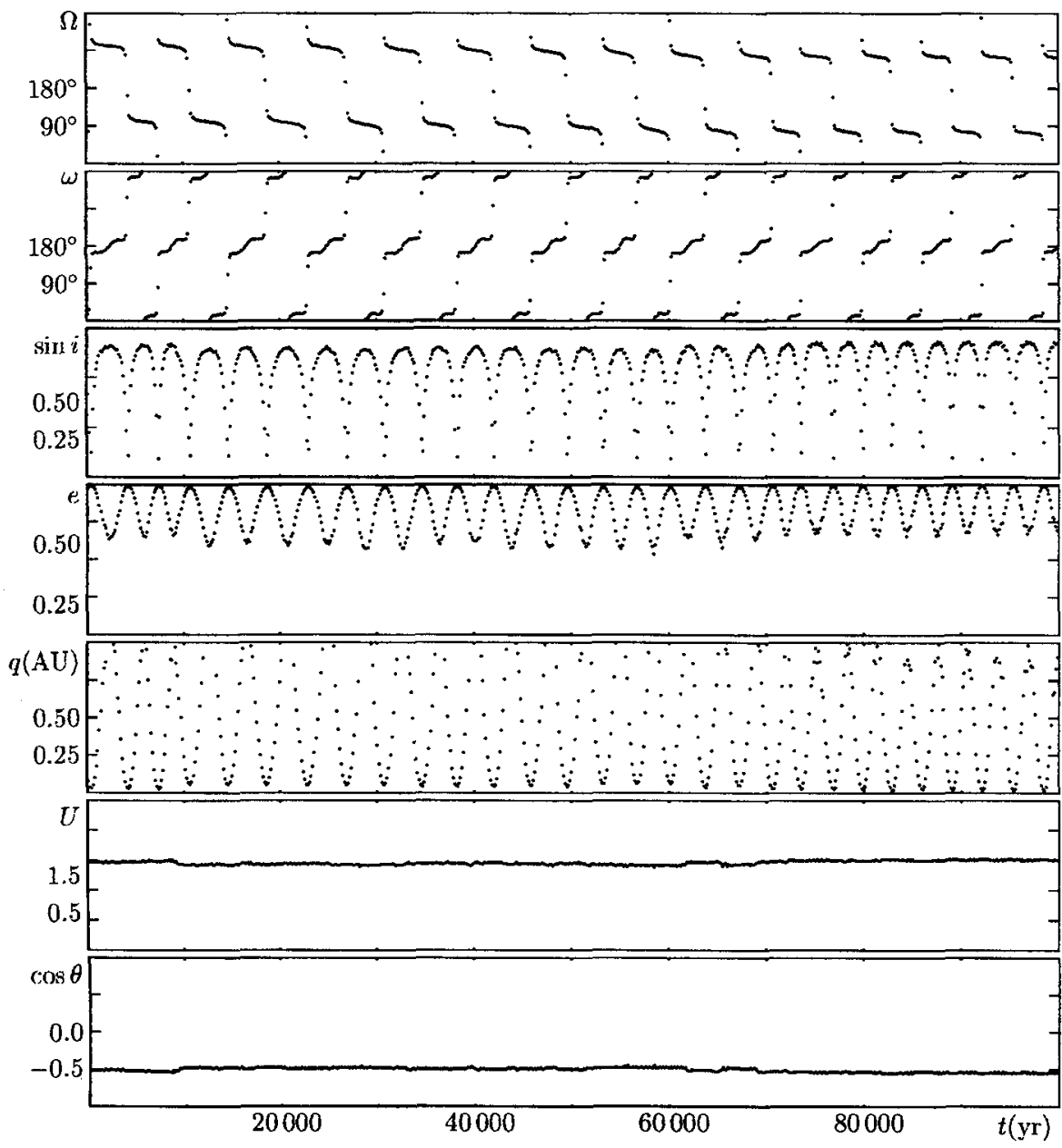

Fig. 1. $100000 \mathrm{yr}$ of evolution of a fictitious comet with an orbit similar to that of 96P/Machholz 1 in a model Solar System composed of the Sun, the Earth and Jupiter on circular, coplanar, not mutually perturbing orbits. The near constancy of the new variables $U$ and $\cos \theta$ is evident.

discussed by Jenniskens et al. 1997, and by Williams and Collander-Brown 1998). The values of $U, \theta$ and $\phi$ for comets and asteroids are computed from the current orbital elements, using Öpik's formulae; note that if the orbit of the comet/asteroid is not currently node-crossing with that of the Earth, the value of $\phi$ computed in this way is different from what it would be at node-crossing, due to the associated changes in $e$ and $i$, and thus is to be considered just as a rough approximation.

Table II gives the streams whose parents have already been suggested in the literature; we have eliminated from the output the many associations that, even to a visual inspection, appear spurious, in order to save space. 
TABLE II

Approximate orbital and geocentric data of some of the streams found by Jopek et al. (1999), together with those of the presumed parent body given in literature, if recovered by our procedure. All quantities are as in Table I, except that $V_{G}$ is substituted by $U=V_{G} / 29.7$, and $\cos \theta$ is given instead of $\theta$. For the parent bodies, the value of $\phi$ in the same quadrant as that of the stream is given; note that this means that two different values of $\phi$ are given for $96 \mathrm{P} / \mathrm{Machholz} 1$, for the two streams to which it has been associated.

\begin{tabular}{|c|c|c|c|c|c|c|c|c|}
\hline Stream name & $q$ & $e$ & $i$ & $\omega$ & $\Omega$ & $\bar{U}$ & $\cos \theta$ & $\phi$ \\
\hline Lyrids & $0 . \overline{92}$ & 0.99 & 80 & 214 & 32 & 1.58 & -0.48 & $19 \overline{8}$ \\
\hline C/1861 G1 Thatcher & 0.92 & 0.98 & 80 & 213 & 32 & 1.58 & -0.48 & 196 \\
\hline Perseids & 0.95 & 0.95 & 113 & 150 & 139 & $1 . \overline{99}$ & -0.75 & 164 \\
\hline 109P/Swift-Tuttle & 0.96 & 0.96 & 113 & 153 & 139 & 2.01 & -0.77 & 168 \\
\hline Taurids (N) & 0.32 & 0.85 & 3 & 299 & 213 & 1.01 & -0.24 & 267 \\
\hline Taurids (S) & 0.34 & 0.82 & 6 & 118 & 27 & 0.94 & -0.24 & 275 \\
\hline 2P/Encke & 0.33 & 0.85 & 12 & 186 & 335 & 1.01 & -0.23 & 250 \\
\hline Quadrantids & 0.98 & 0.68 & 72 & 171 & 282 & 1.38 & -0.44 & 176 \\
\hline 96P/Machholz 1 & 0.13 & 0.96 & 60 & 15 & 95 & 1.47 & -0.51 & 111 \\
\hline Geminids & 0.14 & 0.90 & 24 & 324 & 261 & 1.18 & -0.45 & 258 \\
\hline (3200) Phaethon & 0.14 & 0.89 & 22 & 322 & 266 & 1.12 & -0.47 & 252 \\
\hline Monocerotids & 0.18 & 1.00 & 37 & 129 & 80 & 1.45 & -0.37 & 286 \\
\hline D/1917 F1 Mellish & 0.19 & 0.99 & 33 & 121 & 89 & 1.39 & -0.35 & 290 \\
\hline Leonids & 0.98 & 0.92 & 162 & 173 & 235 & 2.39 & -0.98 & 168 \\
\hline 55P/Tempel-Tuttle & 0.98 & 0.90 & 163 & 173 & 235 & 2.35 & -0.98 & 166 \\
\hline Orionids & 0.57 & 0.97 & 165 & 83 & 29 & 2.22 & -0.91 & 288 \\
\hline S. $\delta$ Aquarids & $0 . \overline{08}$ & 0.97 & 27 & 151 & 308 & 1.38 & -0.47 & 278 \\
\hline 96P/Machholz 1 & 0.13 & 0.96 & 60 & 15 & 95 & 1.47 & -0.51 & 291 \\
\hline N. $\delta$ Aquarids & 0.10 & $0 . \overline{95}$ & 21 & 328 & $\overline{142}$ & $1.2 \overline{8}$ & -0.45 & 262 \\
\hline
\end{tabular}

Our computations did not recover $1 \mathrm{P} / \mathrm{Halley}$ as possible parent body for the Orionids, due to the large difference in longitude of perihelion (about $60^{\circ}$ ), and did not give any candidate parent body for the Northern $\delta$ Aquarids.

We could have included, as candidate parent body of both branches of the Taurids, the recently discovered asteroid $1998 \mathrm{QS}_{52}$, since it is associated to them by our procedure. Its parameters are, in fact, $q=0.31 \mathrm{AU}, e=0.86, i=18^{\circ}$, $\omega=242^{\circ}, \Omega=261^{\circ}, U=1.05, \cos \theta=-0.26$ and $\phi=293^{\circ}$. It must be noted, however, that this asteroid is currently nearly node-crossing, so that its value of $\phi$ cannot be reconciled easily with those of either branch of the Taurids.

Concerning the remaining streams, we present in Table III the results. In the Table are omitted the candidate parent comets of the near-parabolic streams $\varepsilon$ Geminids and $\sigma$ Hydrids, since all of them have longitudes of the node too far away from the stream for being possible parents; also omitted are the candidate parents of the $\kappa$ Cygnids, since they are long-period comets. Of the candidate parents in the Table, to our knowledge only 45P/Honda-Mrkos-Pajdusáková had been previously suggested. We have started to model the evolution of fictitious streams ejected by all of these candidates to check whether they can actually be the parents of the observed streams. 
TABLE III

Same as Table II for the streams found by Jopek et al. (1999) with no presumed parent body given in literature. We have omitted the candidate parents of some streams for the reasons given in the text. Note that our procedure associates $1991 \mathrm{GO}$ only to the Northern branch of the $\varepsilon$ Piscids, and $1998 \mathrm{FW}_{4}$ only to the Southern branch, whereas the three other candidate parents are associated to both branches, and that it associates (8176) 1991 WA to both branches of the $\alpha$ Virginids, but 1998 VP only to the Northern one.

\begin{tabular}{|c|c|c|c|c|c|c|c|c|}
\hline Stream name & $q$ & $e$ & $i$ & $\omega$ & $\Omega$ & $\bar{U}$ & $\cos \theta$ & $\phi$ \\
\hline$\alpha$ Capricornids $(\mathrm{N})$ & 0.58 & 0.78 & 6 & 268 & 134 & 0.77 & 0.02 & 262 \\
\hline D/1783 W1 Pigott & 1.46 & 0.55 & 45 & 355 & 59 & 0.75 & 0.08 & 211 \\
\hline 45P/Honda-Mrkos-Pajd. & 0.53 & 0.82 & 4 & 326 & 89 & 0.84 & -0.02 & 252 \\
\hline (4596) $1981 \mathrm{QB}$ & 1.08 & 0.52 & 37 & 248 & 155 & 0.72 & 0.03 & 196 \\
\hline $1991 \mathrm{VB}$ & 1.32 & 0.41 & 6 & 135 & 257 & 0.71 & 0.09 & 231 \\
\hline$\kappa$ Cygnids & 0.98 & 0.76 & 39 & 202 & 147 & 0.84 & 0.03 & 194 \\
\hline$\varepsilon$ Geminids & 0.81 & 0.96 & 174 & 231 & 203 & 2.36 & -0.97 & 258 \\
\hline$\chi$ Orionids (N) & 0.38 & 0.83 & 3 & 291 & 265 & 0.94 & -0.19 & 267 \\
\hline $1995 \mathrm{UO}_{5}$ & 0.56 & 0.64 & 36 & 151 & 39 & 0.90 & -0.25 & 222 \\
\hline 199500 & 0.48 & 0.78 & 24 & 211 & 350 & 0.92 & -0.17 & 235 \\
\hline$\chi$ Orionids (S) & 0.51 & 0.79 & 5 & 96 & $7 \overline{7}$ & 0.84 & -0.05 & 276 \\
\hline $1998 \mathrm{VD}_{31}$ & 0.52 & 0.80 & 10 & 113 & 48 & 0.84 & -0.05 & 296 \\
\hline $1997 \mathrm{VM}_{4}$ & 0.49 & 0.81 & 14 & 124 & 46 & 0.89 & -0.10 & 299 \\
\hline $1979 \mathrm{XB}$ & 0.65 & 0.71 & 25 & 76 & 86 & 0.80 & -0.05 & 316 \\
\hline$\sigma$ Hydrids & 0.24 & 0.98 & 126 & 122 & $\overline{78}$ & 1.95 & -0.73 & 295 \\
\hline$\alpha$ Virginids $(\mathbf{N})$ & 0.41 & 0.83 & 8 & 288 & 34 & 0.94 & -0.16 & 263 \\
\hline$\alpha$ Virginids (S) & 0.32 & 0.87 & 7 & 118 & 198 & 1.04 & -0.22 & 275 \\
\hline (8176) $1991 \mathrm{WA}$ & 0.56 & 0.64 & 40 & 242 & 67 & 0.94 & -0.28 & 319 \\
\hline 1998 VP & 1.01 & 0.46 & 44 & 101 & 226 & 0.85 & -0.15 & 186 \\
\hline$\varepsilon$ Piscids $(\mathrm{N})$ & 0.58 & 0.76 & 5 & 268 & 190 & 0.74 & 0.02 & 263 \\
\hline$\varepsilon$ Piscids (S) & 0.61 & 0.73 & 4 & 85 & 5 & 0.71 & 0.03 & 276 \\
\hline $1991 \mathrm{GO}$ & 0.66 & 0.66 & 10 & 89 & 25 & 0.65 & 0.05 & 235 \\
\hline $1998 \mathrm{FW}_{4}$ & 0.68 & 0.73 & 4 & 75 & 3 & 0.66 & 0.12 & 292 \\
\hline $1998 \mathrm{HL}_{49}$ & 0.63 & 0.64 & 11 & 239 & 207 & 0.65 & 0.00 & 306 \\
\hline $1997 \mathrm{YR}_{10}$ & 1.15 & 0.33 & 37 & 191 & 270 & 0.66 & -0.01 & 341 \\
\hline 1991 YA & 1.53 & 0.44 & 44 & 174 & 274 & 0.71 & 0.09 & 329 \\
\hline
\end{tabular}

\section{Conclusions}

The geocentric variables, directly linked to observed quantities, proposed by Valsecchi et al. (1999) lead to a stream identification criterion rather different from the classical one by Southworth and Hawkins (1963); the new criterion has been successfully tested on photographic meteor orbits by Jopek et al. (1999), with results disagreeing from those obtained with Southworth and Hawkins' criterion in the case of near-ecliptical streams.

We have exploited in this paper the secular near-invariance of some of the new variables to look for candidate parent bodies for the streams found with the new criterion; we recover all the known parents except 1P/Halley for the Orionids, and find some not previously suggested candidate parents for several of the remaining streams. 


\section{References}

Babadzhanov,P.B., Obrubov, Yu.V.: 1993, Dynamics and relationships between interplanetary bodies I. Machholz and its meteor showers, in Meteoroids and their parent bodies, J. Štohl and I.P. Williams, eds., Astronomical Institute of the Slov. Acad. Sci., Bratislava, Slovakia, 49-52

Carusi, A., Valsecchi, G.B., Greenberg, R.: 1990, Planetary close encounters: geometry of approach and post-encounter orbital parameters, Cel. Mech. \& Dyn. Astro., 49, 111

Drummond, J.: 1981, A test of comet and meteor shower associations, Icarus, 45, 545-553

Jenniskens, P., Betlem, H., de Lignie, M., Langbroek, M. and van Vliet, M.: 1997, Meteor stream activity V. The Quadrantids, a very young stream, $A \& A, 327,1242-1252$

Jopek, T.: 1993, Remarks on the meteor orbital similarity D-criterion, Icarus, 106, 603-607

Jopek, T.J., Froeschlé, Cl.: 1997, A\&A, 320, 631-641

Jopek, TJ., Valsecchi, G.B., Froeschlé, Cl.: 1999, Meteor streams identification: a new approach. II. Application to 865 photographic meteor orbits, MNRAS, in press

Kozai, Y., 1962, Secular perturbations of asteroids with high inclination and eccentricity, AJ, 67, 591-598

Lindblad, B.A.: 1971, Smithson. Contr. Astrophys., 12, 1-13

Öpik, E.J.: 1976, Interplanetary Encounters, Elsevier, New York

Southworth, R.B., Hawkins, G.S.: 1963, Statistics of meteor streams, Smithson. Contr: Astrophys., 7, 261-285

Thomas, F., Morbidelli, A., 1996, The Kozai resonance in the outer solar system and the dynamics of long-period comets, Cel. Mech. \& Dyn. Astro., 64, 209-229

Valsecchi, G.B., Jopek, T.J., Froeschlé, Cl.: 1999, Meteoroid streams identification: a new approach. I. Theory, MNRAS, in press

Williams, I.P., Collander-Brown, S.J., 1998 The parent of the Quadrantid meteoroid stream, MNRAS, 294, 127-138 